\title{
New catalogue of optically visible open clusters and candidates ${ }^{\star}$
}

\author{
W. S. Dias ${ }^{1}$, B. S. Alessi ${ }^{1}$, A. Moitinho ${ }^{2}$, and J. R. D. Lépine ${ }^{1}$ \\ 1 Universidade de São Paulo, Dept. de Astronomia, CP 3386, São Paulo 01060-970, Brazil \\ 2 Observatorio Astronómico Nacional, UNAM, Apdo. Postal 877, CP 22800, Ensenada B.C., México
}

Received 14 March 2002 / Accepted 15 April 2002

\begin{abstract}
We have compiled a new catalogue of open clusters in the Galaxy which updates the previous catalogues of Lyngå (1987) and of Mermilliod (1995) (included in the WEBDA database). New objects and new data, in particular, data on kinematics (proper motions) that were not present in the old catalogues, have been included. Virtually all the clusters (1537) presently known were included, which represents an increment of about 347 objects relative to the Lyngå (1987) catalogue. The catalogue is presented in a single table containing all the important data, which makes it easy to use. The catalogue can be accessed on line either at http://www.astro.iag.usp.br/ ${ }^{\sim}$ wilton/ or as an electronic table which will be made available at the CDS.
\end{abstract}

Key words. galaxy: open clusters and associations: general - catalogs

\section{Introduction}

In this work, we introduce a new catalogue of the open clusters of our Galaxy. Open clusters have long been recognized as important tools to investigate the kinematics of star formation regions, aspects of Galactic structure such as the location of spiral arms, Galactic dynamics, or even the chemical abundance gradients in the disk.

With the publication of the Hipparcos Catalogue (ESA 1997) and its sub-products, the Tycho (ESA 1997) and Tycho2 (Høg et al. 2000) catalogues, and with individual work using CCDs for photometry and/or astrometry, we have seen a large growth of the available data on open clusters in a short time.

Among the recent results, we note the discovery of new open clusters by different authors: Platais et al. (1998) discovered 12 new objects using Hipparcos data, Chereul et al. (1999) discovered 3 new probable loose open clusters, and Dutra \& Bica (2001) discovered 42 objects at infra-red wavelengths using the 2MASS survey. Important contributions were given by Baumgardt et al. (2000) and Dias et al. (2001, 2002) who determined the mean proper motions of more than a hundred clusters, using the Hipparcos and Tycho2 catalogues, respectively. Dias et al. (2001, 2002) also computed the membership probabilities of the stars in the cluster fields. Other recent results are the deter-

\footnotetext{
Send offprint requests to: W. S. Dias, e-mail: wilton@usp.br

* Tables 1a, 1b, 2 and 3 are only available in electronic form at http://www.astro.iag.usp.br/ ${ }^{\sim}$ wilton/, or at the CDS via anonymous ftp to cdsarc.u-strasbg.fr (130.79.128.5) or via

http://cdsweb.u-strasbg.fr/cgi-bin/qcat?J/A+A/389/871
}

mination of the fundamental parameters of 423 clusters by Loktin et al. (2000) and the discussion of the problem of the differences in the distances obtained with parallaxes and by photometric main sequence fitting (see Pinsonneault et al. 1998, and references therein). The latest publications on open clusters are divulged in the SCYON electronic newsletter hosted by the University of Heidelberg $^{1}$ in parallel with the WEBDA database ${ }^{2}$.

Most of the basic data, as well as other results, are included in the WEBDA database (Mermilliod 1995), which is the most complete open cluster database presently available. The WEBDA database includes not only the data contained in the Lynga (1987) catalogue, which is also a basic reference much used in the literature, but also provides a huge amount of additional information. Most of this information is, however, presented in separate files, available individually for each cluster. Also, the database is not updated with recently discovered clusters and new designations proposed in the literature (as discussed in next section). Therefore, the main reasons that prompted us to prepare a new catalogue, instead of simply adding newly discovered objects, were the need to have the relevant information in a single file, for ease of use, and more important, the fact that the previous catalogues do not provide the open clusters' proper motions and radial velocities in a systematic way.

In this work, we inserted the available information on open clusters' fundamental parameters, kinematics and metalicity in a single file. We believe that this list will

\footnotetext{
${ }^{1}$ http://www.rzuser.uni-heidelberg.de/ s17/scyon/ current.html

${ }^{2}$ http://obswww. unige.ch/webda/
} 
be an important tool for all types of research on open clusters. Section 2 describes the contents of the catalogue and the main reference sources. In Sect. 3 we comment on the new data included.

\section{The catalogue}

In this new open cluster catalogue we used previous ones such as the WEBDA, ESO Catalogue (Lauberts 1982) and Lyngå (1987) as a starting point. The basic data contained in these catalogues are coordinates, age, apparent diameter, colour excess, and distance. We inserted new objects, and when available, kinematical and metalicity data. We made extensive use of the Simbad database and of the literature to find data on the clusters or on individual stars of the clusters, to obtain radial velocities and proper motions averaged over a number of stars. We do not claim, however, that the catalogue is the result of a complete survey of all the bibliography on open clusters.

Our catalogue (Table 1a) consists of a single list of fundamental parameters and kinematical data, with bibliographic notes. The file is self-explanatory and fully documented internally. The present version of the catalogue includes information for 1537 open clusters. For each cluster we list its equatorial coordinates in J2000.0 and the following parameters, when available: angular apparent diameter; distance; colour excess; age; mean proper motions and errors; number of stars used in the proper motion computation and references; mean radial velocity and error; number of stars used in the radial velocity determination and references; mean metalicity and errors; number of stars used in the metalicity determination. An identical list (Table $1 \mathrm{~b}$ ) is also provided with positions and proper motions in galactic coordinates. The full bibliographic references are given in a separate file (Table 2).

In total, $94.7 \%$ of the objects have estimates of their apparent diameters, and $37 \%$ have distance, $E(B-V)$ and age determinations. Concerning the data on kinematics, $18 \%$ have their mean proper motions listed, $12 \%$ their mean radial velocities, and $9 \%$ have both information simultaneously.

Many objects in the list were visually checked in the Digitized Sky Survey ${ }^{3}$ (DSS) plates, and in several cases the central coordinates of the clusters were corrected. This is the case of clusters like Lynga 8, Stock 12, Stock 15 and vdB-Hagen 164, just to mention a few, that present great differences in position.

Throughout our visual inspection of the DSS plates, there were also many cases in which no cluster could be found (e.g. several Ruprecht, Collinder and Loden clusters), even in large fields around their catalogued coordinates. They were nevertheless kept in the catalogue, but a comment was added. We shall refer to these "objects" as "non-identified clusters". Among these, are the NGC objects flagged as "non-existent" in the The Revised New General Catalogue of Non stellar Astronomical

\footnotetext{
${ }^{3}$ http://archive.stsci.edu/dss/
}

Objects (RNGC) (Sulentic \& Tifft 1973). On the other hand, some clusters noted as "non-existent" in the RNGC seem to be actual clusters (e.g. NGC 2017, NGC 2609, NGC 3036, NGC 5800, NGC 6115, NGC 6444). These have been marked as "recovered" in our catalogue.

A complementary table (Table 3 ) of the clusters with available photometric data was also built. Table 3 consists of four columns: cluster name; bands observed with CCD; bands observed with photomultipiers; bands observed with photographic plates. For each cluster, only bands with more than ten observed stars are listed. At the present, the table only lists the $U B V R I$ bands, but it will be extended to other commonly used photometric systems (e.g. uvby $\beta$, Geneva, Washington, Vilnius, etc.). The data table was assembled using data collected from WEBDA and from searches in the literature.

\section{Comments on new information and new data included}

In this section we comment on some important information given in the catalogue.

Designations - An additional remark on the nature of the open clusters is provided. Among others, we flag the POCR (Possible Open Cluster Remnant, Bica et al. 2001). There are 34 objects located at relatively high galactic latitudes $\left(b \geq 15^{\circ}\right)$ which appear to be late stages of star cluster dynamical evolution. The categories also include possible moving groups like the objects catalogued by Latysev and non-identified clusters.

Kinematics - Recently, many open clusters were investigated and their mean proper motions (Dias et al. 2001, 2002; Baumgardt et al. 2000) could be determined. New mean proper motions for 280 objects, and radial velocities for 182 were inserted in the list.

Fundamental parameters - The main source of the fundamental parameters (reddening, distance and age) was the WEBDA which uses the information compiled by Lyngå (1987), Loktin et al. (2000), Dambis (1998) and Malysheva (1997).

All the clusters investigated by Baumgardt et al. (2000) had their distances estimated from the mean Hipparcos parallaxes of the stars considered as members. Recently we investigated 4 open clusters and determined the mean Hipparcos parallax of stars with membership probability provided by Tycho 2 proper motions (Dias et al. 2001). The catalogue includes distances derived from mean parallaxes for Ruprecht 147, Stock 10, vdB-Hagen 23, vdB-Hagen 34, all within $1 \mathrm{kpc}$. Also, a number of parameters from isolated studies were added.

Newly discovered open clusters - The list includes 191 clusters not present in the previous catalogues. To mention some cases: Platais et al. (1998) - 12 open clusters were discovered using Hipparcos data. They are nearby and extended objects; ESO-SC - these objects (more than 100) were published as probable new open clusters in the ESO catalogue Lauberts (1982); Loiano 1 A photometric study of the surrounding stellar field 
(Bernabei \& Polcaro 2001) revealed that this object lies inside the sky area of a previously undetected open cluster of intermediate age. Alessi 1 to 12 are non catalogued objects in the solar vicinity. Their fundamental parameters were recently determined showing that they are located at $d \leq 1 \mathrm{kpc}$ (Alessi et al. 2002).

\section{Summary and conclusions}

We have presented a new list of open clusters containing revised data compiled from old catalogues and from isolated papers recently published. This catalogue (Table 1a) has been developed mainly to be an efficient tool for open cluster studies since it presents all the available basic data (fundamental parameters and kinematics) in a single easy-to-use list. The catalogue is regularly updated, and the latest version is available at http://www.astro.iag.usp.br/ ${ }^{\sim}$ wilton/. An alternative list (Table $1 \mathrm{~b}$ ) with positions and proper motions in galactic coordinates is also made available. Since it is expected that the catalogue will be used in the selection of observational targets, an additional table of open clusters with available photometric data (Table 3 ) is also provided. Finally, Table 2 includes the references to the data used in Tables $1 \mathrm{a}$ and $1 \mathrm{~b}$.

In this edition, 1537 objects are given, of which 356 are not given in the catalogue compiled by Lyngå (1987). The new objects include 191 open cluster published in the literature, and 11 recently discovered open cluster with fundamental parameters determined by our group and yet unpublished.

Nearly all the clusters $(94.7 \%)$ have estimates of their apparent diameters. Distances, $E(B-V)$ and ages are listed for $37 \%$. Concerning the data on kinematics, $18 \%$ have mean proper motions determinations, $12 \%$ mean radial velocities, and $9 \%$ have both information simultaneously. These results point out to the observers that a large effort is still needed to improve the data on kinematics. Our group is presently working in this direction.

Acknowledgements. We use data from Digitized Sky Survey which were produced at the Space Telescope Science Institute under U.S. Government grant NAG W-2166. The images of these surveys are based on photographic data obtained using the Oschin Schmidt Telescope on Palomar Mountain and the UK Schmidt Telescope. Extensive use has been made of the Simbad and WEBDA databases. This project was supported by FAPESP (grant number 99/11781-4).

\section{References}

Alessi, B. S., Moitinho, A., \& Dias, W. S. 2002, in preparation Baumgardt, H., Dettbarn, C., \& Wielen, R. 2000, A\&AS, 146, 251

Bernabei, S., \& Polcaro, V. F. 2001, A\&A, 371, 123

Bica, E., Santiago, B. X., Dutra, C. M., et al. 2001, A\&A, 366, 827

Chereul, E., Crézé, M., \& Bienaymé, O. 1999, A\&AS, 135, 5

Dambis, A. K. 1998, Astro. Lett., 25, 10

Dias, W. S., Lépine, J. R. D., \& Alessi, B. S. 2001, A\&A, 376, 441

Dias, W. S., Lépine, J. R. D., \& Alessi, B. S. 2002, A\&A, 388, 168

Dutra, C. M., \& Bica, E. 2001, A\&A, 376, 434

ESA 1997, The Hipparcos and Tycho Catalogues (European Space Agency, ESA)

Høg, E., Fabricius, C., Makarov, V. V., et al. 2000, A\&A, 355, $\mathrm{L} 27$

Lauberts, A. 1982, ESO/Uppsala survey of the ESO(B) atlas (Garching: European Southern Observatory, ESO)

Loktin, A. V., Gerasimenko, T. P., \& Malisheva, T. 2000, Astro. Astrophys. Trans., in press

Lyngå, G. 1987, Computer Based Catalogue of Open Cluster Data, 5th ed. (Strasbourg: CDS)

Malysheva, L. K. 1997, Astro. Lett., 23, 585

Mermilliod, J. C. 1995, in Information and On-Line Data in Astronomy, ed. D. Egret, \& M. A. Albrecht (Dordrecht: Kluwer), 127

Pinsonneault, M. H., Stauffer, J., Soderblom, D. R., King, J. R., \& Hanson, R. B. 1998, ApJ, 504, 170

Platais, I., Kozhurina-Platais, V., \& van Leuwen, F. 1998, AJ, 116,2423

Sulentic, J. W., \& Tifft, W. G. 1973, The Revised New General Catalogue of Nonstellar Astronomical Objects (Tucson: U. of Arizona Press) 